\title{
A hierarchy of coupled maps
}

\author{
Neil J. Balmforth \\ Department of Applied Mathematics and Statistics, University of California, Santa Cruz, California 95064 \\ Antonello Provenzale \\ ISAC-CNR, Corso Fiume 4, 10133 Torino, Italy and ISI Foundation, Torino, Italy \\ Roberto Sassi \\ Department of Applied Mathematics and Statistics, University of California, Santa Cruz, California 95064
}

(Received 13 February 2002; accepted 1 July 2002; published 22 August 2002)

\begin{abstract}
A large number of logistic maps are coupled together as a mathematical metaphor for complex natural systems with hierarchical organization. The elementary maps are first collected into globally coupled lattices. These lattices are then coupled together in a hierarchical way to form a system with many degrees of freedom. We summarize the behavior of the individual blocks, and then explore the dynamics of the hierarchy. We offer some ideas that guide our understanding of this type of system. (C) 2002 American Institute of Physics. [DOI: 10.1063/1.1502929]
\end{abstract}

Ensembles of low-order dynamical systems are often used to model the interaction, competition, and synchronization among dynamically coupled, but distinct, objects. In this genre, coupled map lattices are one of the simplest and widely used objects. Despite their simplicity, coupled maps show a wide range of behavior, both with global coupling (in which each map is affected by the dynamics of all other maps; a mean field interaction) and local coupling (where each map interacts only with its nearest neighbors). This richness is one reason for their popularity. Another reason is their computational simplicity; temporal evolution amounts to a straightforward sequence of algebraic operations. In the current article, we take a conceptual step beyond the idea of a simple ensemble of maps; we consider ensembles of ensembles of maps, a lattice of lattices, coupled in a hierarchical way. The underlying idea is that our hierarchy is a mathematical metaphor for a complicated entity composed of an organization with many levels and scales. In the study of this vast dynamical system, we try to make sense of the possible range of dynamical behavior of which that system is capable.

\section{INTRODUCTION}

Many natural systems are composed of a large number of interacting elements, some of which are strongly coupled to each other, while others are only weakly bound together. Often, these elements are composed of smaller subunits, which are, in turn, composed of still smaller sub-subunits, and so on down to a basic, perhaps molecular, level. This structure can take the form of a hierarchy of basic constituents wherein a rich network of units acts as a single, albeit complex, entity. Classic examples of this type of organization include such things as living or social organisms, the climate, and pictures of turbulence as a hierarchy of interacting eddies.

In this work, we explore some of the dynamics of such a hierarchy of interacting elements. To build our metaphor, we begin at the lowest "level" with a collection of map lattices. Each lattice is a globally coupled network of maps, and is independent of the other lattices. The lattices are then coupled to maps on a level "above," which are themselves organized into further lattices or groups. These groups are coupled to maps on the next higher level, which again are collected into another group. The repetition of this construction creates a hierarchy of many levels; every level consists of a lattice of maps, each with connections to the maps on the same level, to the lattices on the level beneath and to a map above. At the head of the hierarchy is a single presiding map. This leads us to contemplate a system with a very large number of degrees of freedom; a typical system contains $10^{6}$ or more map elements. We use logistic maps as the fundamental elements, and so this translates to as many dimensions.

Although the mathematical constructions considered here are motivated by the behavior of natural systems with hierarchical structure, our considerations are not meant to provide an understanding of those entities. Our goal is more of a mathematical game-we simply pose the question that, given such a complicated system, can we make some systematic deductions concerning its dynamical capabilities? Some more specific questions we address are: How does the hierarchy beneath influence the dynamics of the presiding map? Can disorganized behavior at the lower levels be organized in the upper levels? Vice versa, can organized motion on the lower levels be destroyed as we go from the bottom to the head of the tree? Is the self-similar structure of the hierarchy imprinted on the dynamics? All these questions are motivated by the issues that one often faces when considering turbulent fluids (with their cascade of motion through an inertial range of length and time scales), interpreting time series of physical and biological phenomena (with the everpresent sources of noise and error, which are really highdimensional deterministic systems), and in thinking of the thermodynamical principles governing vast ensembles of in- 
teracting elements (where one assumes notions of equipartition and equilibrium in order to construct statistical ensembles).

\section{FORMULATION}

\section{A. The elementary unit}

We construct the hierarchy from a basic element. This element, or building "block" as we shall refer to it, is composed of a lattice of coupled maps that interact through their mean field. In addition, the mean field of the lattice is also channelled into another map, the "node," which does not influence the underlying lattice.

The equations for the block are

$$
\begin{array}{ll}
\text { Node: } & X_{n+1}=(1-\rho) f\left(X_{n}\right)+\rho M_{n}, \\
\text { Lattice: } & x_{n+1}^{j}=(1-\epsilon) f\left(x_{n}^{j}\right)+\epsilon M_{n},
\end{array}
$$

where

$$
M_{n}=\frac{1}{N} \sum_{j=1}^{N} f\left(x_{n}^{j}\right)
$$

is the lattice's mean field. The coupling strengths are measured by the parameters, $\epsilon$ and $\rho$, which range from zero to unity, with the additional constraint $0 \leqslant \epsilon+\rho \leqslant 1 ; \epsilon$ measures the strength of the coupling inside the lattice, while $\rho$ measures the strength of the coupling between the lattice and the node. We further take logistic maps, with

$$
f(x)=1-a x^{2}
$$

and $0<a \leqslant 2$. $^{1}$

Thus, the building block is composed of a coupled map lattice of the kind explored by Kaneko and others ${ }^{2-4}$ (we refer to the latter article as paper I) that is fed into another logistic map. An understanding of the dynamics of this system is a necessary ingredient in the exploration of the full hierarchy, so we give a crude summary in the next section.

\section{B. The hierarchy}

The system of interest in this study is a lattice of $\left(N^{L}\right.$ $-1) /(N-1)$ maps, structured on an $L$-level tree and hierarchically coupled. The hierarchy begins at the lowest level, labeled $l=L$, then progresses through $L$ stages or levels up to the apex, a single map labeled $l=1$ (the "head"). On every level $l, N^{l-1}$ maps are collected in $N^{l-2}$ groups of $N$ maps each. Maps in each block are coupled together through their mean fields. The scheme is illustrated in Fig. 1.

The lattice evolution is expressed mathematically by the system,

$$
\begin{aligned}
& l=1 \quad x_{n+1}^{[1 ; 1 ;-]}=(1-\rho) f\left(x_{n}^{[1 ; 1 ;-]}\right)+\rho M_{n}^{[2 ; 1]}, \\
& l \neq 1, l \neq L \quad x_{n+1}^{[l ; m ; g]}=(1-\epsilon-\rho) f\left(x_{n}^{[l ; m ; g]}\right)+\epsilon M_{n}^{[l ; g]} \\
& +\rho M_{n}^{[l+1 ; m+N(g-1)]}, \\
& l=L \quad x_{n+1}^{[L ; m ; g]}=(1-\epsilon) f\left(x_{n}^{[L ; m ; g]}\right)+\epsilon M_{n}^{[L ; g]},
\end{aligned}
$$

where
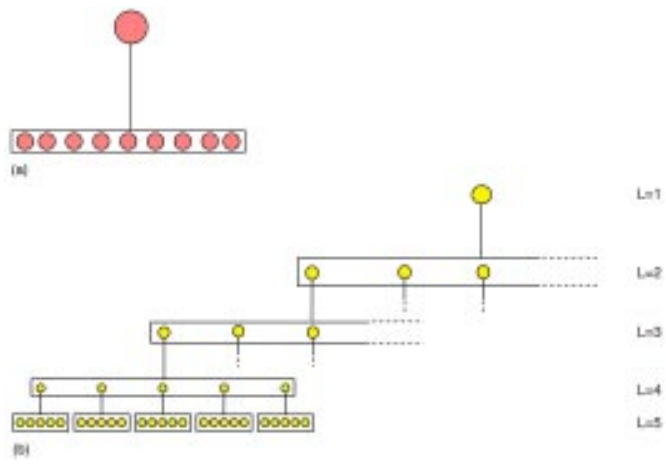

FIG. 1. (Color online) Schematic illustration of (a) the elementary units (here consisting of a node connected to a lattice of 9 maps), and (b) the hierarchy constructed from them (here assuming $N=L=5$ ).

$$
M_{n}^{[l ; g]}=\frac{1}{N} \sum_{j=1}^{N} f\left(x_{n}^{[l ; j ; g]}\right)
$$

is now the group mean field. In the notation used above, the state of an individual map is indicated by $x_{n}^{[l ; m ; g]}$, and it contributes to the mean field, $M_{n}^{[l ; g]}$, of the group of which it is a member. The subscript $n$ indicates time (or iteration number, since time is an integer). The first superscript, $l$, indicates the level and runs from 1 to $L$; the second superscript, $m$, labels individual maps on the same level, with $1 \leqslant m \leqslant N$, and the third superscript, $g$, indicates the group to which the map belongs on a given level, with $1 \leqslant g \leqslant N^{l-2}$. For the head, $l=1$, there are no groups and we use the symbol " - " instead of the group number.

The parameter $\epsilon$ takes the same meaning as before, namely the coupling strength among maps within each lattice or group. Now, however, $\rho$ measures the coupling between levels.

\section{DYNAMICS OF THE BUILDING BLOCK}

\section{A. The lattice}

The dynamics of an individual lattice is documented in earlier papers. We summarize the results of these articles in Figs. 2-4. Very roughly, the behavior of the lattice falls into one of three categories: synchronization, clustering, and incoherence. In the first category, the lattice evolves into a completely synchronized state in which every element executes the same trajectory (an orbit of the logistic map). In the clustering state, the lattice divides itself into synchronized families; within each family, the maps execute the same orbit, but the different families follow different orbits. In the third state, each element follows an orbit that is almost independent of the others, and the lattice has little correlation. Figures 2 and 3 show regime diagrams for the three possible phases, and Fig. 4 provides examples that illustrate them. In Figs. 2 and 3, the dashed line shows the rough border between incoherence and clustered lattices, which is relatively well defined. This border can be constructed using the mean-field theory described later in Sec. IV, and discussed also in paper I. The second curve in these pictures is based on further results from paper I; to the right of this curve we expect only synchronized lattices (the curve com- 


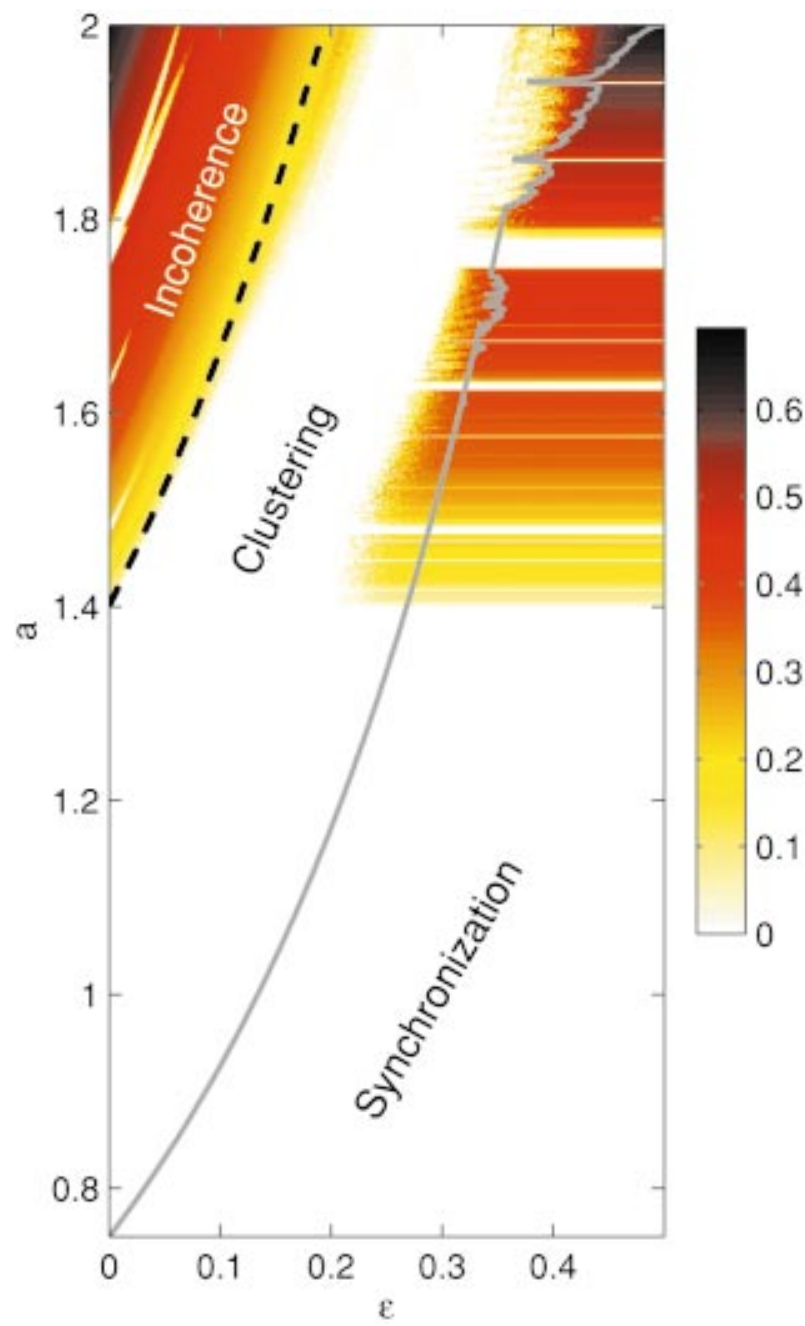

FIG. 2. (Color) A regime diagram indicating roughly where a single map lattice synchronizes, clusters or is incoherent. The plot shows the average, leading Lyapunov exponent of the lattice, determined from ten computations with lattices of 100 elements. Each lattice was iterated 11000 times, and the first 1000 iterations were discarded. The dashed line shows the approximate border between clustering and incoherence, and is constructed using the mean-field theory of Sec. IV. The border between clustering and synchronization is far less well defined and there is significant overlap of the two regimes. Based on the considerations of paper I (and, in particular, Fig. 1 of that article), we expect that there are only synchronized lattices to the right of the second curve.

bines the stability boundary of the fully synchronized lattice with that of the most stable cluster state-an equally partitioned cluster of two families executing an asynchronous period-2 orbit-whichever lies furthest to the right). However, the boundary between synchrony and clustering is more ambiguous and there is substantial overlap of the two phases.

Because they follow orbits of the quadratic map, the dynamics of a synchronized lattice is solely a function of $a$ and is dictated by the familiar bifurcation diagram of that map. For $a<0.75$, there is a stable fixed point; if $0.75<a$ $<a_{\infty} \approx 1.40115519$, there are periodic solutions; and when $a>a_{\infty}$, the orbit is chaotic except inside windows of $a$ containing stable periodic orbits. Cluster states are more often periodic or quasiperiodic than chaotic, ${ }^{3,4}$ and the dynamics of an incoherent lattice is, by definition, high dimensional chaos. We bring out these features in Fig. 2, which shows the

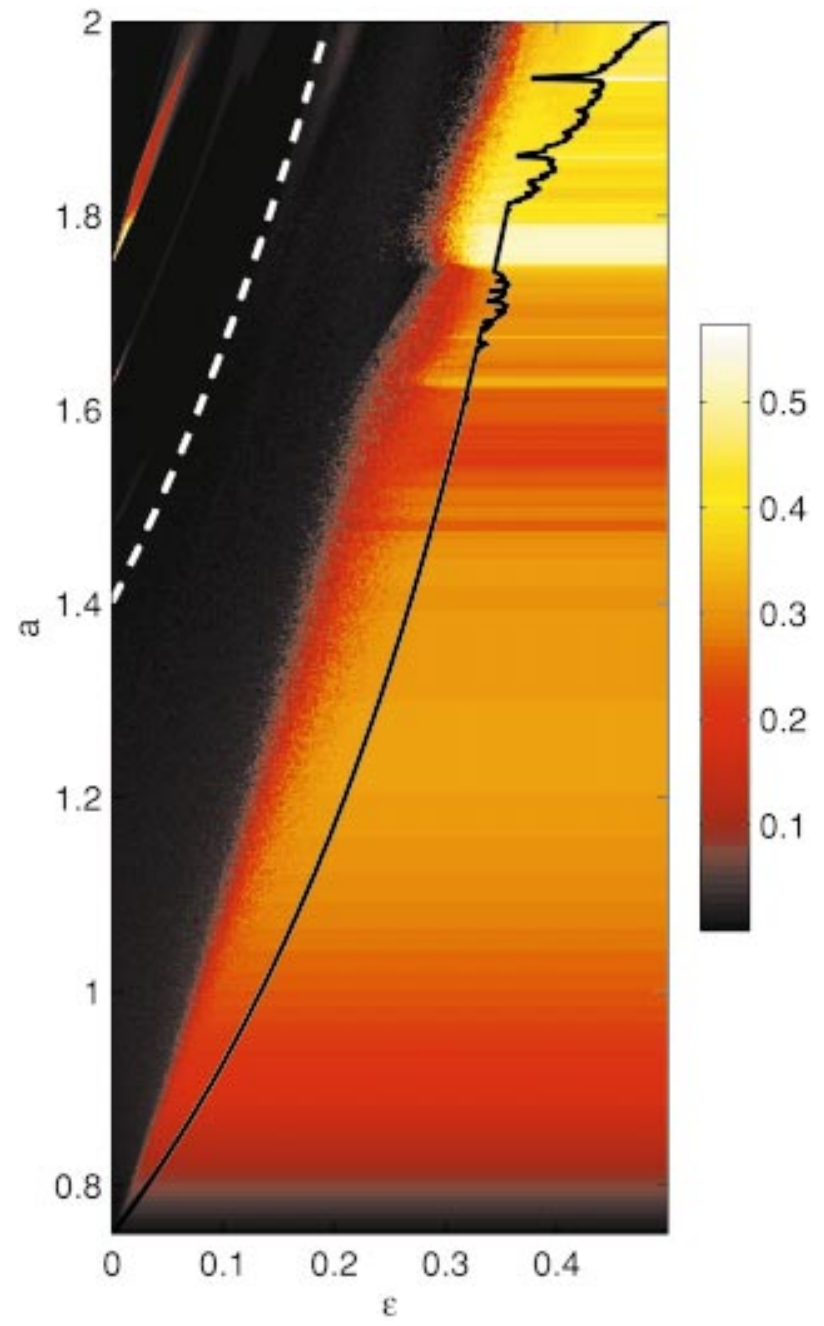

FIG. 3. (Color) A second regime diagram for the behavior of a single map lattice. The plot now shows the fluctuation level of the mean field (the average standard deviation over an orbit of length 10000 , determined from ten computations with lattices of 100 elements; each lattice was iterated 11000 times, and the first 1000 iterations were discarded). As in Fig. 2, the two curves show the rough border between incoherent and clustered lattices, and the rightmost limit of lattices that are not synchronized.

leading Lyapunov exponent of the lattice. The onset of incoherence is relatively clearly seen in this picture, but there are regimes in which clustered and synchronized lattices are not distinguished.

The transition between synchronization and clustering is better highlighted in Fig. 3, which shows the fluctuation level of the mean field (as given by the standard deviation of a long time series of the mean field, averaged over ten realizations of the lattice). Synchronized lattices have fluctuation levels dictated by that of a single map, and as $a$ increases, this level can become quite large. On the other hand, the fluctuation level is typically the smallest for incoherent lattices because each map evolves largely independently and so the fluctuations decay like $N^{-1}$. A significant fraction of the clustered lattices also have a reduced fluctuation level. This arises because there are typically a large number of cluster states with a wide array of different "partitions" (that is, the number of families and the way the maps are distributed amongst them). By chance alone, clusters that have a more 
(a) $\varepsilon=0.1$

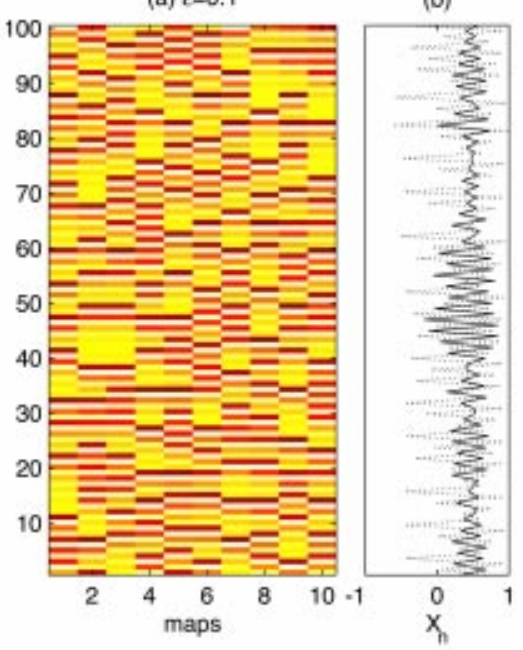

(c) $\varepsilon=0.2$

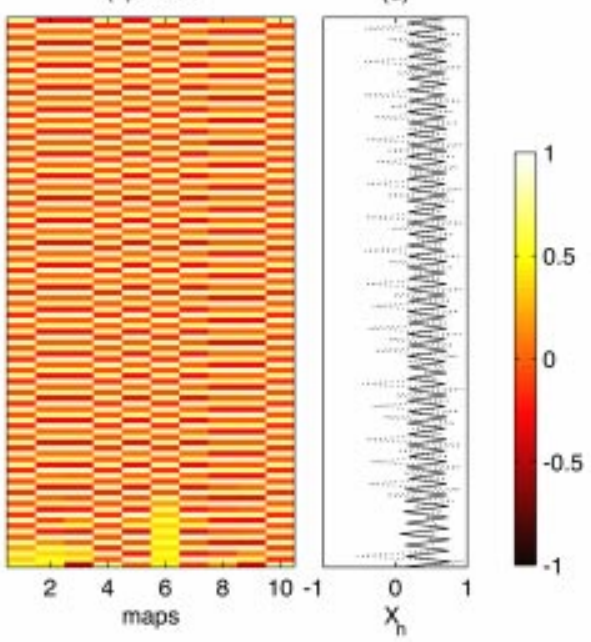

(e) $\varepsilon=0.4$

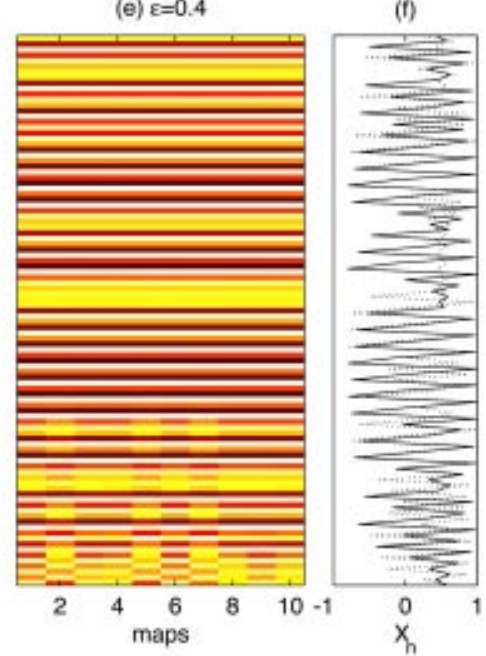

FIG. 4. (Color online) Evolution of sample blocks with $N=10$ and $a=1.8$. The map index is on the abscissa and time runs on the ordinates. Three pairs of panels are shown. In each pair, the left-hand picture illustrates the evolution of the lattice (the key to the coloring is given in the color bar). The right-hand plot in each pair displays the evolution of the corresponding node. In the latter, two computations are shown: the dotted line has $\rho=0.1$, and the solid line shows $\rho=0.5$. Panels (a)-(b) are for $\epsilon=0.1$ (an incoherent lattice), (c) -(d) are for $\epsilon=0.2$ (a clustered lattice), and (e)-(f) are for $\epsilon=0.4$ (a synchronized lattice).

uniform partition (an equal distribution of maps in the families) are encountered more often than clusters with very unequal partitions; these more common clusters have reduced mean field fluctuations because families typically evolve out of step with one another (that is, the mean field contributions from each cluster tend to offset one another). A departure from this rule of thumb occurs in a strip of Fig. 3 located just to the left of the black curve. Here, most of the clustered lattices have unequal partitions and their mean fields are dominated by the larger family, which renders this field more like that of a synchronized lattice. These lattices also are usually chaotic, rather than periodic like their more equally partitioned relatives, and for this reason, the strip also appears in Fig. 2. Thus, the borders suggested in Figs. 2 and 3 do not separate clustering and synchrony but more properly the end of equally partitioned clusters.

\section{B. The node}

The dynamics of the node is determined by the competition between the intrinsic dynamics of the map and the mean-field forcing from the underlying lattice. The strength of the forcing is measured by the coupling parameter $\rho$. For smaller values of $\rho$, coupling is weak and the node evolves largely under its own dynamics; depending on the value of $a$, we then expect periodic or chaotic dynamics. For larger $\rho$ on the other hand, the intrinsic dynamics of the node plays a secondary role, and we expect that the node dynamics reflects that of the forcing. This effect is obvious in the sample blocks shown in Fig. 4-the node's dynamics is largely independent of $\epsilon$ (and hence the state in which the underlying lattice falls) for $\rho=0.1$, but not when $\rho=0.5$. For example, in panels $(\mathrm{c})-(\mathrm{d})$, the node is chaotic for $\rho=0.1$ but has the same periodicity as the lattice for $\rho=0.5$.

If the uncoupled nodal map is chaotic $\left(a>a_{\infty}\right)$, the transition from independent to slaved dynamics is traced by the
Lyapunov exponent of the node; Fig. 5 displays such exponents on the $(a, \rho)$ plane. In panel (a), $\epsilon=0.1$, and (depending on the value of $a$ ) the underlying lattice is either clustered or incoherent. The exponent is positive for small $\rho$ and $a>a_{\infty}$, implying independent evolution of the node. As $\rho$ increases, the exponent typically decreases and becomes negative, whereafter we interpret that the node is slaved to the forcing. For $\epsilon=0.1$, this passage of the exponent is interrupted by sharp transitions through skewed windows of stable periodic orbits.

The second panel of the figure shows computations with $\epsilon=0.6$, for which the underlying lattice is fully synchronized (and so the precise values of $\epsilon$ and $N$ are irrelevant). A similar picture of the node dynamics emerges, although the structure in panel (b) is more complicated. In this case, periodicorbit windows are horizontal and the exponent occasionally becomes positive even inside these windows. This indicates that there are parameter regimes in which both the synchronized lattice and an uncoupled node are periodic, but the coupled node is chaotic.

The curves also drawn in Fig. 5 are constructed as follows: When the underlying lattice is not synchronized, as illustrated in Fig. 3, the mean field fluctuates at a low level. Consequently, the main effect of the lattice is to provide a nearly constant forcing, $M(a, \epsilon)$, and so

$X_{n+1} \approx(1-\rho) f\left(X_{n}\right)+\rho M=(1-\rho)\left(1-a X_{n}^{2}\right)+\rho M$.

A simple rescaling recasts this equation in the form of the quadratic map with a new parameter,

$$
\widetilde{a}=a(1-\rho)(1-\rho+\rho M) .
$$

In other words, the coupling of the node to the underlying lattice renormalizes (and in fact reduces) the effective map parameter (the notion of renormalization was used in paper I, and will recur later when we consider the hierarchy; see also Ref. 5). Moreover, because $a_{\infty}$ is the value of $\widetilde{a}$ for which the 
(a) $\varepsilon=0.1$

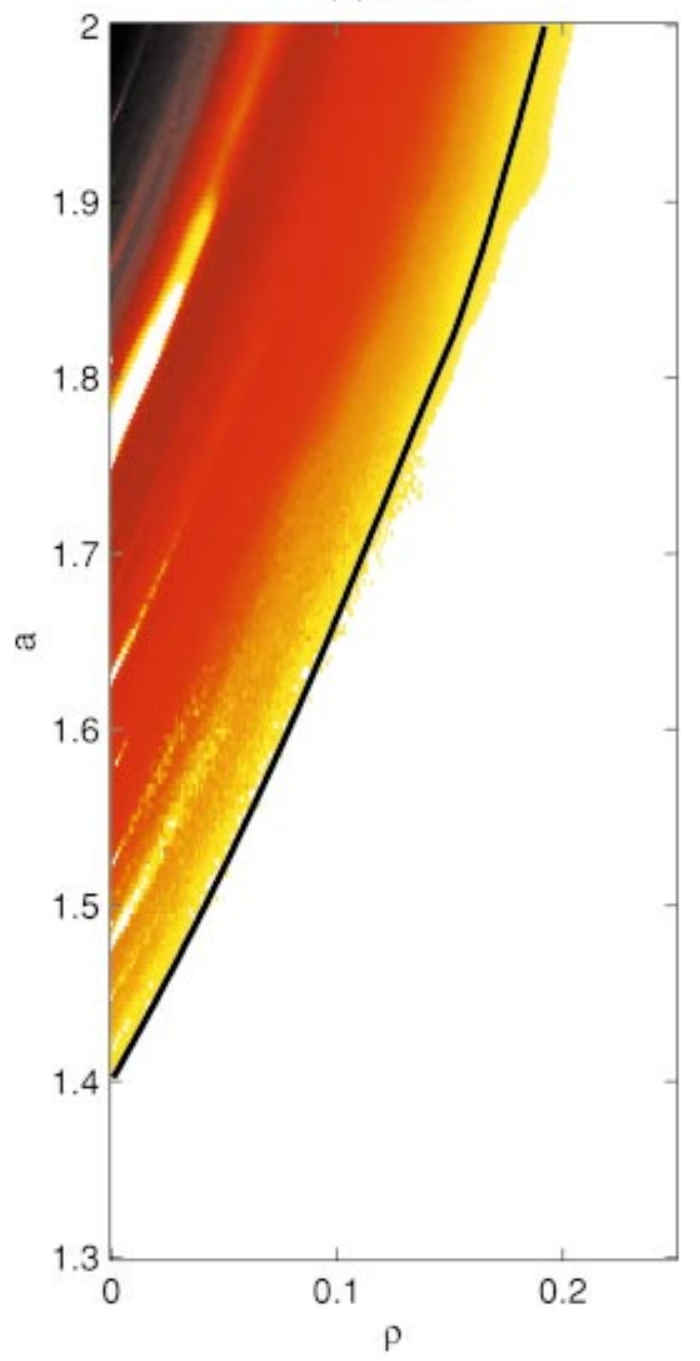

(b) $\varepsilon=0.6$

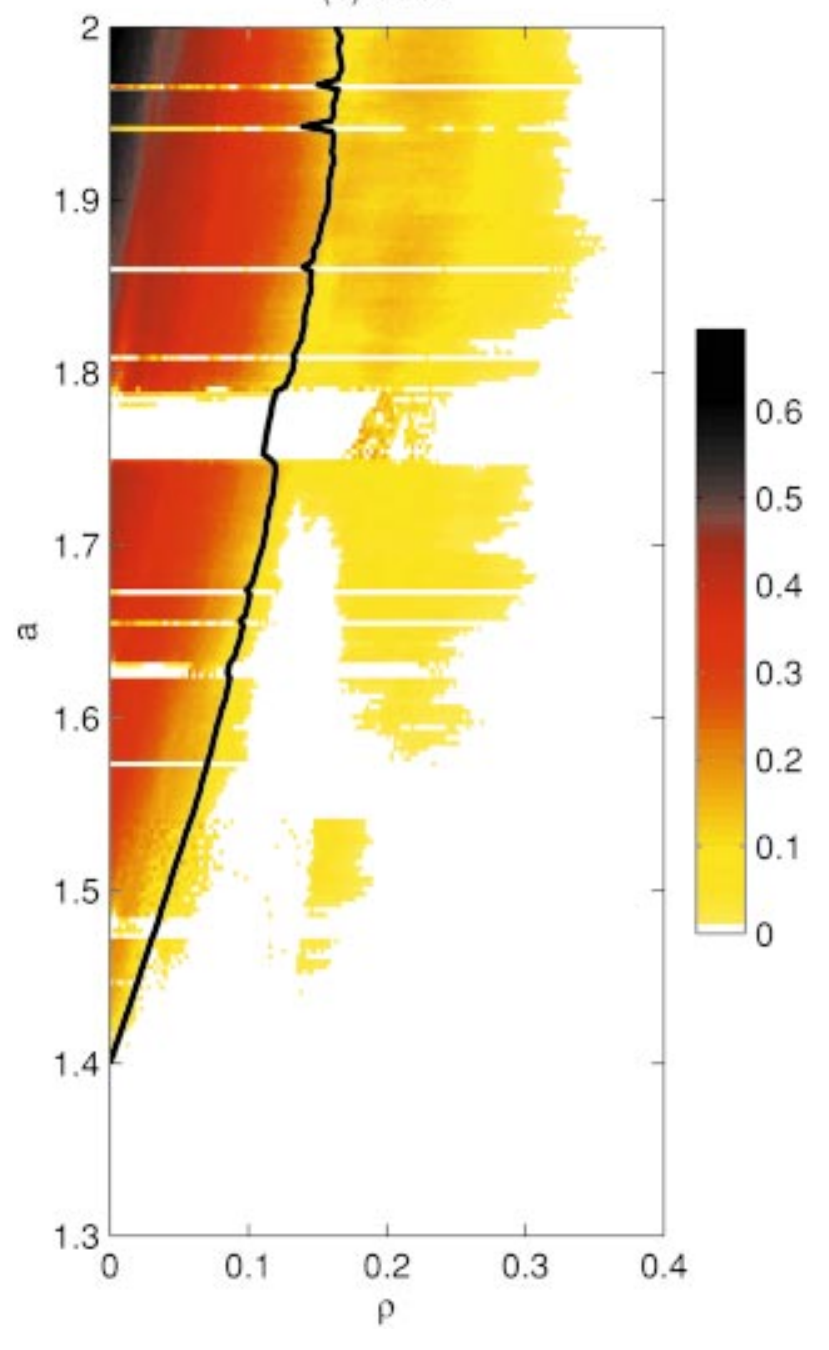

FIG. 5. (Color) Lyapunov exponent of the node of blocks with varying $a$ and $\rho$, for (a) $\epsilon=0.1$ and (b) $\epsilon=0.6$; $N=100$. The computational length is 6000 iterations, of which the first 1000 are discarded in order to remove transients. The curve is obtained from (8); for panel (b) the value of the mean field used is a time average.

Lyapunov exponent of the renormalized map first becomes positive, the node must become slaved to the underlying lattice when $\widetilde{a} \approx a_{\infty}$, or

$$
a_{\infty} \approx a(1-\rho)(1-\rho+\rho M),
$$

which, given $a$, can be solved immediately for $\rho$. This curve compares well with the numerical results (see Fig. 5).

The edges of the skewed windows of periodic orbits in Fig. 5(a) can be traced in a similar way. Also, despite the fact that the underlying lattice is synchronized (and so the mean field fluctuations are not small), curve (8) also provides an estimate of the location of the transition in panel (b); the mean field, $M$, used in this case is a temporal average over the synchronized orbit.

\section{HIERARCHY DYNAMICS}

We begin a discussion of the dynamics of the hierarchy by describing two useful concepts that help to navigate our path through the rich phenomenology: frustration and meanfield renormalization (in two forms). Rather than embark on a detailed and systematic discussion of the dynamical behavior (which would have limited success and utility given the huge number of degrees of freedom involved), we then propose a number of guiding principles to help organize how we may think of the hierarchy. For illustration, we choose $N$ $=10$ for the number of maps in each block; many of the results are similar on using other values of $N$, but we offer some remarks later on how the map-number per block affects the dynamics.

\section{A. Frustration}

In some ways, the dynamics of the hierarchy can be thought of as resulting from a persistent frustration. Each map evolves under the competition between its own dynamics, the synchronizing effect of the mean field of the elements within the same block, and the forcing field driving the element from the level below. In certain limits of the parameters, one of these competing effects can dominate, but in many others, the competition frustrates the hierarchy. 
One solution of the equations is the fully synchronized hierarchy, $x_{n}^{[\cdot ; \cdot ; \cdot]}=X_{n}$. For a single map lattice, the analog of this solution plays an important role (see the previous section). But the synchronized hierarchy is of secondary interest. This is because on any one level, the blocks evolve independently of one another. Hence, there is no way to synchronize the driving signals entering the maps on the level above, and so those maps can never synchronize. True synchronization can only be achieved if all the driving signals at each level are identical. This is assured if all the blocks fall into the same orbit, which would require both stability and a favorable initial condition. However, it is straightforward to show that fully synchronized hierarchies are linearly stable only if the synchronized orbit is not chaotic $\left(a<a_{\infty}\right.$, or inside windows of stable periodic orbits). Moreover, with a large number of levels in the hierarchy and maps within each block, the probability that the hierarchy will find a periodic synchronized state from an arbitrary initial condition is essentially zero (each map must fall into the same phase of the cycle) ${ }^{6}$

Despite the lack of true synchronization, it is possible to achieve a weaker form of synchronization on a level by increasing the global coupling $\epsilon$. The enhanced coupling can then counter the desynchronizing effect of the varying driving signals, and the differences between the maps on the level are thereby relegated to small fluctuations. We christen this kind of dynamics, "imperfect synchronization." Note that, because arbitrarily small desynchronizing perturbations can lead to order unity fluctuations around chaotic synchronized states, ${ }^{7}$ chaotic, imperfect synchronous orbits should typically undergo episodic desynchronizations.

For similar reasons, variations in driving signals also prevent true clustering within the hierarchy; "imperfect" clustering arises instead. As for the coupled map lattice, these latter states are often periodic. In fact, because clustered states often have reduced mean-field fluctuations (Fig. 3 ), the variations in driving signals can be relatively small. In turn, this means that imperfect clustering can be pronounced, as we will see in the examples later in this section.

\section{B. Level renormalization}

If the mean fields at one level have low-amplitude fluctuations, we can extend the ideas of renormalization used in Sec. III to the lattice immediately above: We set $M_{n}^{[l+1 ; g]}$ $\approx \mu^{l+1}$; the maps on level $l$ then evolve according to

$$
\begin{aligned}
x_{n+1}^{[l ; m ; g]} \approx & (1-\epsilon-\rho)\left[1-a\left(x_{n}^{[l ; m ; g]}\right)^{2}\right] \\
& +\frac{\epsilon}{N} \sum_{j=1}^{N}\left[1-a\left(x_{n}^{[l ; j ; g]}\right)^{2}\right]+\rho \mu^{l+1} .
\end{aligned}
$$

On making the change of variable, $y_{n}^{[l ; m ; g]}=x_{n}^{[l ; m ; g]} /[1-\rho$ $\left.+\rho \mu^{l+1}\right]$, we recast (9) in the form,

$y_{n+1}^{[l ; m ; g]} \approx(1-\widetilde{\boldsymbol{\epsilon}})\left[1-\widetilde{a}\left(y_{n}^{[l ; m ; g]}\right)^{2}\right]+\frac{\widetilde{\boldsymbol{\epsilon}}}{N} \sum_{j=1}^{N}\left[1-\widetilde{a}\left(y_{n}^{[l ; j ; g]}\right)^{2}\right]$,

with

$$
\widetilde{a}=a(1-\rho)\left(1-\rho+\rho \mu^{l+1}\right), \quad \widetilde{\epsilon}=\frac{\epsilon}{1-\rho} .
$$

That is, each group at the $l$ th-level behaves as a globally coupled map lattice with renormalized map and coupling parameters.

The parameter $\widetilde{a}$ decreases with $\rho$ while $\widetilde{\epsilon}$ increases. This has the interesting consequence that the lowest level can be incoherent but the level immediately above can be clustered or synchronized, at least in a mean sense, when the point $(\widetilde{\epsilon}, \widetilde{a})$ falls below the regime of incoherent lattices. The renormalized level can no longer be considered incoherent in this eventuality, and so the nature of the dynamics of the levels yet higher is unclear.

\section{Complete renormalization}

It is also possible to make a complete renormalization of the entire hierarchy when the mean fields at every level have low-level fluctuations. In this instance, the whole system is incoherent, and we fix $M_{n}^{[l ; g]} \approx \mu^{l}$ at each level. The result, after similar manipulations is a logistic map describing every element in the hierarchy,

$$
z_{n+1}^{[l ; m ; g]} \approx 1-\check{a}^{l}\left(z_{n}^{[l ; m ; g]}\right)^{2},
$$

with $z_{n}^{[l ; m ; g]}=x_{n}^{[l ; m ; g]} /\left(1-\epsilon-\rho+\epsilon \mu^{l}+\rho \mu^{l+1}\right)$ and the renormalized map parameter,

$\check{a}^{l}= \begin{cases}a(1-\rho)\left(1-\rho+\rho \mu^{l+1}\right) & l=1 \\ a(1-\rho-\epsilon)\left(1-\rho-\epsilon+\rho \mu^{l+1}+\epsilon \mu^{l}\right) & l=2, \ldots, L-1 \\ a(1-\epsilon)\left(1-\epsilon+\epsilon \mu^{l}\right) & l=L .\end{cases}$

This formula for $\check{a}^{l}$ can also be obtained from a further reduction of (10), and is complicated by the presence of $\mu^{l}$, which itself depends on $\check{a}^{l}$. We can proceed a little further by noting that the mean field of the renormalized element is given by

$$
Z\left(\check{a}^{l}\right)=1-\frac{1}{N} \check{a}^{l} \sum_{m}\left(z_{n}^{[l ; m ; g]}\right)^{2},
$$

and depends upon $\check{a}^{l}$ alone. By expressing $z_{n}^{[l ; m ; g]}$ in terms of $x_{n}^{[l ; m ; g]}$, we may write the right-hand side of this expression in terms of $\mu^{l}$. On eliminating that mean field, we are finally lead to

$$
\check{a}^{l}\left[1-\rho-\epsilon Z\left(\check{a}^{l}\right)\right]=a(1-\epsilon-\rho)^{2}\left(1-\rho+\rho \mu^{l+1}\right),
$$

$l \in[2, L-1]$, which is an implicit equation for $\check{a}^{l}$. At the lowest level, $\check{a}^{L}\left[1-\epsilon Z\left(\check{a}^{L}\right)\right]=a(1-\epsilon)^{2}$, while the presiding map is governed by (7).

To solve the implicit equation, we rearrange the expression by dividing by the factor $1-\rho-\epsilon Z\left(\check{a}^{l}\right)$. Given an initial first guess for $\check{a}^{l}$, we compute $Z\left(\check{a}^{l}\right)$, recalculate $\check{a}^{l}$ from the implicit equation, and then iterate until the variation of $\breve{a}^{l}$ is less than about $10^{-4}$. A sample solution obtained in this way for a seven-level hierarchy is shown in Fig. 6. Although, the iteration appears to converge up to this tolerance in this (and many other) calculations, there are hidden problems. For a generic value of the map parameter, it is known that statistical averages such as $Z\left(\check{a}^{l}\right)$ are not continuous func- 
(a)

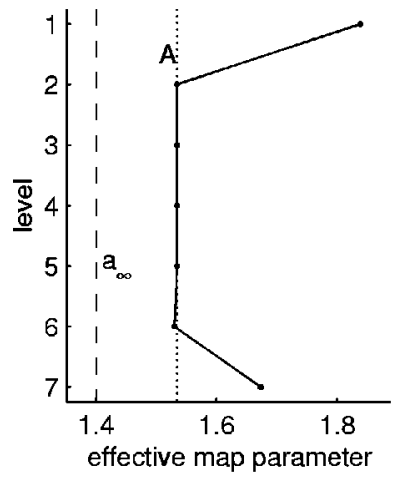

(b)

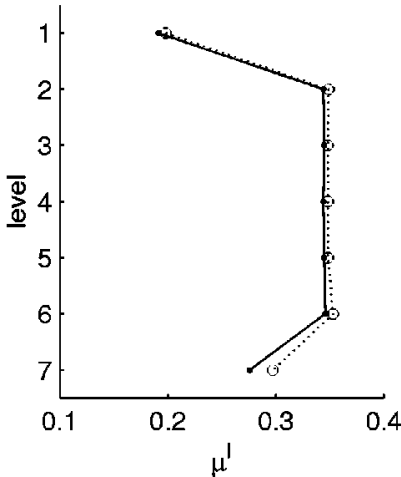

(c)

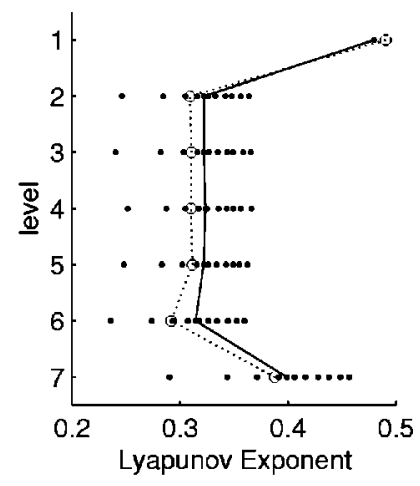

FIG. 6. Renormalizing the hierarchy I. Panel (a) shows the variation of the effective map parameter, $\check{a}^{l}$, with level, $l$, for $a=2, \epsilon=0.1, \rho=0.05$ and $N$ $=10$. In panels (b) and (c), we show the corresponding mean fields, $\mu^{l}$, and Lyapunov exponents (the circles joined by dotted lines). These are compared with data computed directly from a single block at each level of a full hierarchy with the same values of $a, \epsilon$ and $\rho$. In panel (b), the hierarchy mean fields are shown by points connected by solid lines. In (c), the dots show the ten Lyapunov exponents [obtained via Gram-Schmidt orthogonalization (Ref. 8)] for the selected block; the continuous line connects their average values. The parameters values are the same of Fig. 8 (left).

tions because of the windows of stable periodic orbits. Moreover, even with regularization by numerical resolution, $Z\left(\check{a}^{l}\right)$ still varies wildly with $\check{a}^{l}$. Therefore, the implicit equation is unlikely to have a unique solution. Of course, if the renormalized map enters a window of a stable periodic orbit, it cannot be considered incoherent, and so the presence of such windows signifies an inconsistency in the theory. In reality, the renormalized map is in a state of continual agitation from the mean-field fluctuations. This noisiness may well both remove the stable periodic orbit windows and regularize the computation of $\check{a}^{l}$. However, such considerations require an extension of the theory, which is beyond our current discussion. Instead we are content to iterate the equation for $\check{a}^{l}$ to the required tolerance and hope all is well. The comparison of calculations like that shown in Fig. 6 with results from a full hierarchy (see below) gives us some confidence that this hope is not completely unfounded.

In the example of Fig. 6, the renormalized parameter decreases from $l=L$ to $l=L-1$, recovers slightly from $l$ $=L-1$ to $l=L-2$, and then asymptotes to a common value, $\check{a}^{l} \rightarrow A$, given implicitly by

$$
A=\frac{a(1-\epsilon-\rho)^{2}}{1-(\epsilon+\rho) Z(A)} .
$$

Only the mean field of the lowest level contributes for $l=L$, whereas the elements at any other level experience mean fields from both their own level and the one immediately below. Consequently, the renormalization is least at $l$ $=L$ and $l=1$, and, in general, is greatest at $l=L-1$.

Also shown in the figure are the corresponding mean fields, $\mu^{l}$, and the Lyapunov exponents of the renormalized maps. These are compared with data computed directly from a single block at each level of a full hierarchy with the same base parameters. As indicated by the figure, there is agreement between the mean fields, and also the Lyapunov exponents, once we average the $N(=10)$ exponents of a single block. This comparison gives support both to the mean-field theory outlined in this section, and also to the idea that the hierarchy is incoherent.
Now, for complete renormalization of an incoherent hierarchy, all the maps must be chaotic. Hence, $\check{a}^{l}>a_{\infty}$, or

$$
a(1-\rho-\epsilon)\left(1-\rho-\epsilon+\rho \mu^{l+1}+\epsilon \mu^{l}\right)>a_{\infty},
$$

for every $l \in[2, L-1]$. This condition is the generalization of that used in paper I to locate where a single coupled map lattice desynchronizes. Because the renormalization is typically largest at $l=L-1, \check{a}^{L-1}$ is the smallest renormalized parameter, and the condition that the lattice is incoherent becomes

$$
\check{a}^{L-1}=\frac{a(1-\epsilon-\rho)^{2}\left(1-\rho+\rho \mu^{L}\right)}{\left[1-\rho-\epsilon Z\left(\check{a}^{L-1}\right)\right]}>a_{\infty} .
$$

The limiting condition [in which we replace the inequality by an equality and set $\left.Z\left(\check{a}^{L-1}\right)=Z\left(a_{\infty}\right) \approx 0.3761\right]$ can be written as an equation for $\rho$ that is straightforward to solve in terms of $\check{a}^{L}$ [given $\check{a}^{L}$ we can compute $\mu^{L} \equiv(1$ $-\epsilon) Z\left(\check{a}^{L}\right) /\left[1-\epsilon Z\left(\check{a}^{L}\right)\right]$ and $a=\check{a}^{L}\left[1-\epsilon Z\left(\breve{a}^{L}\right)\right] /(1-\epsilon)^{2}$ without solving any implicit equations].

A simpler approximation follows if we only require that $A>a_{\infty}$. This leads to the limiting condition,

$$
a=\frac{a_{\infty}\left[1-(\epsilon+\rho) Z\left(a_{\infty}\right)\right]}{(1-\epsilon-\rho)^{2}},
$$

which depends on the coupling parameters solely through their sum, and can be solved for that combination. We show the two surfaces in the $(\epsilon, \rho, a)$ parameter space that describe these conditions in Fig. 7.

\section{Guidelines}

We now provide some illustrations of the behavior of the hierarchy. To quantify the dynamics, we use two measures: The first is the average squared difference of all the elements in each block $g$ at the $l$ th level, averaged over $\Pi=1000$ iterations,

$D^{[l ; g]}=\frac{1}{\Pi} \sum_{n=1}^{\Pi} \frac{1}{N(N-1)} \sum_{j} \sum_{k}\left(x_{n}^{[l ; j ; g]}-x_{n}^{[l ; k ; g]}\right)^{2}$. 
(a)

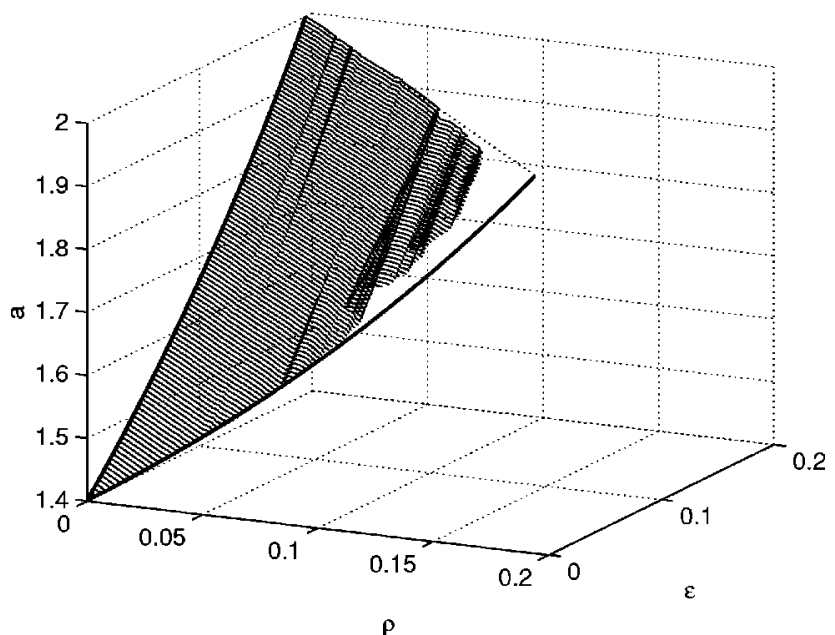

(b)

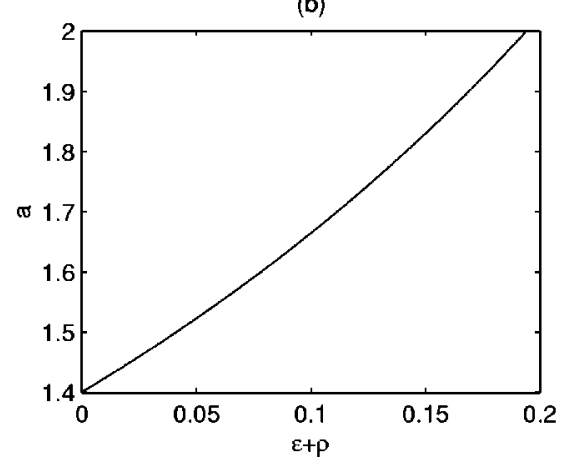

FIG. 7. Renormalizing the hierarchy II. Panel (a) shows the surface in the $(\epsilon, \rho, a)$ parameter space corresponding to the condition in (18), above which we expect the system to be fully incoherent. Panel (b) shows the simpler condition in (19); this condition, for $\rho=0$ is shown earlier in Figs. $2-3$. The continuous lines in panel (a) mark the intersections of this second cylinder with the planes $\epsilon=0$ and $\rho=0$ for comparison with the first surface. The crinkles in the surface in (a) result from the appearance of windows of stable periodic orbits in the renormalized map; as that map should be incoherent (and therefore chaotic), there is an inconsistency in the theory here that must be resolved by explicit consideration of mean-field fluctuations.

This quantity measures the degree of coherence of each coupled lattice in two ways. First, the value of $D^{[l ; g]}$ provides an estimate of the uniformity of an individual block; low values indicate a high degree of synchrony, and incoherent blocks have high values. Second, the spread of values of $D^{[l ; g]}$ on a particular level gives a sense of the range of different kinds of blocks. Closely clustered blocks typically occur with a wide variety, and so the spread of values of $D^{[l ; g]}$ highlights imperfect clusters.

The other measurement is the leading Lyapunov exponent of each group which, because of the hierarchical structure, can be computed for each group in isolation of the others. This exponent indicates the degree of temporal complexity at a particular level; if positive, the level can be considered to evolve independently of the level below. If negative, the exponent reveals either periodic motion or a level slaved to that below.

(1) $(\epsilon, a)$ in the incoherent regime: The lowest level is incoherent and, as a result, feeds a mean field forcing with low-level fluctuations into the level above. Consequently, the upper levels approximately consist of globally coupled map lattices with renormalized parameters. If the renormalization is sufficient to place $(\widetilde{\boldsymbol{\epsilon}}, \widetilde{a})$ out of the incoherent regime, then the upper levels show some degree of clustering and/or synchronization. Otherwise the entire hierarchy is incoherent.

Examples are shown in Fig. 8 for $a=2$ and $\epsilon=0.1$, with $\rho=0.05$ and 0.2. For $\rho=0.05$, according to the theory of Sec. IV B, the renormalized lattice parameters above the lowest level remain in the incoherent regime $(\widetilde{a} \approx 1.8$ and $\widetilde{\epsilon}$ $\approx 0.11$ at level $L-1$ ), and the upper levels are incoherent. This is also consistent with the conditions for incoherence derived in Sec. IVC (see also Fig. 6). For $\rho=0.2$, on the other hand, the renormalized parameters at $l=L-1$ lie in the clustering regime ( $\widetilde{a} \approx 1.3$ and $\widetilde{\epsilon}=0.125$ ), and the higher levels begin to organize.

The lower panels of the figure show our quantitative measures of the dynamics. For the incoherent lattice, the squared differences $D^{[l ; g]}$ fall off slightly on moving up the hierarchy, but otherwise remain at a relatively high level, reflecting the lattice disorder. The Lyapunov exponents also illustrate the chaotic incoherence. In the case with larger vertical coupling, the emergence of some order on the lattice above the lowest level is evident in $D^{[l ; g]}$. The Lyapunov exponents show that, at the $l=6$ level the lattices are slaved to the lower-level dynamics for $\rho=0.2$. However, this situation reverses at the levels above. It seems impossible to extract any trend of the dynamics with level in this second case; there is certainly no self-similarity.

(2) $(\epsilon, a)$ in the clustering regime: If $\rho$ is small $(<\epsilon)$, imperfect clusters exist at the lowest levels. For larger $\rho$ $(>\epsilon)$, the clustering quality degrades because of the increasingly desynchronizing effect of the forcing fields from the level below. At higher $\rho$, imperfect cluster states can again occur if the forcing fields from the level below can be grouped into similar signals. True clustering occurs if there are only a small number of periodic clusters at the lowest level. Then elements on the next level up can receive identical forcing mean fields, and cluster together as a result. True clustering can continue in this way some distance up the hierarchy, but because the number of possible clusters and their phases rapidly multiplies on escalating through the hierarchy, the chance of finding such true clusters quickly declines.

The dynamics is illustrated in Fig. 9 for $a=1.7$ and $\epsilon$ $=0.2$. In the case with $\rho=0.05$, we see the degradation of the clustering quality over the lowest level as we escalate through levels; for $\rho=0.5$, persistent clustering occurs through accidental coincidences of the forcing fields. As indicated by the Lyapunov exponents, there are chaotic signals entering the hierarchy at the lowest level for both hierarchies. Therefore, despite the appearance of the top picture in the second case, both presiding maps are chaotic. The apex for $\rho=0.5$ appears to be nearly periodic because that presiding map receives a chaotic driving signal that has been filtered to low amplitude by the intermediate levels.

The degradation of the cluster quality is also reflected in the decline of the squared differences, $D^{[l ; g]}$, with level, $l$ (roughly speaking, incoherent solutions have the highest 

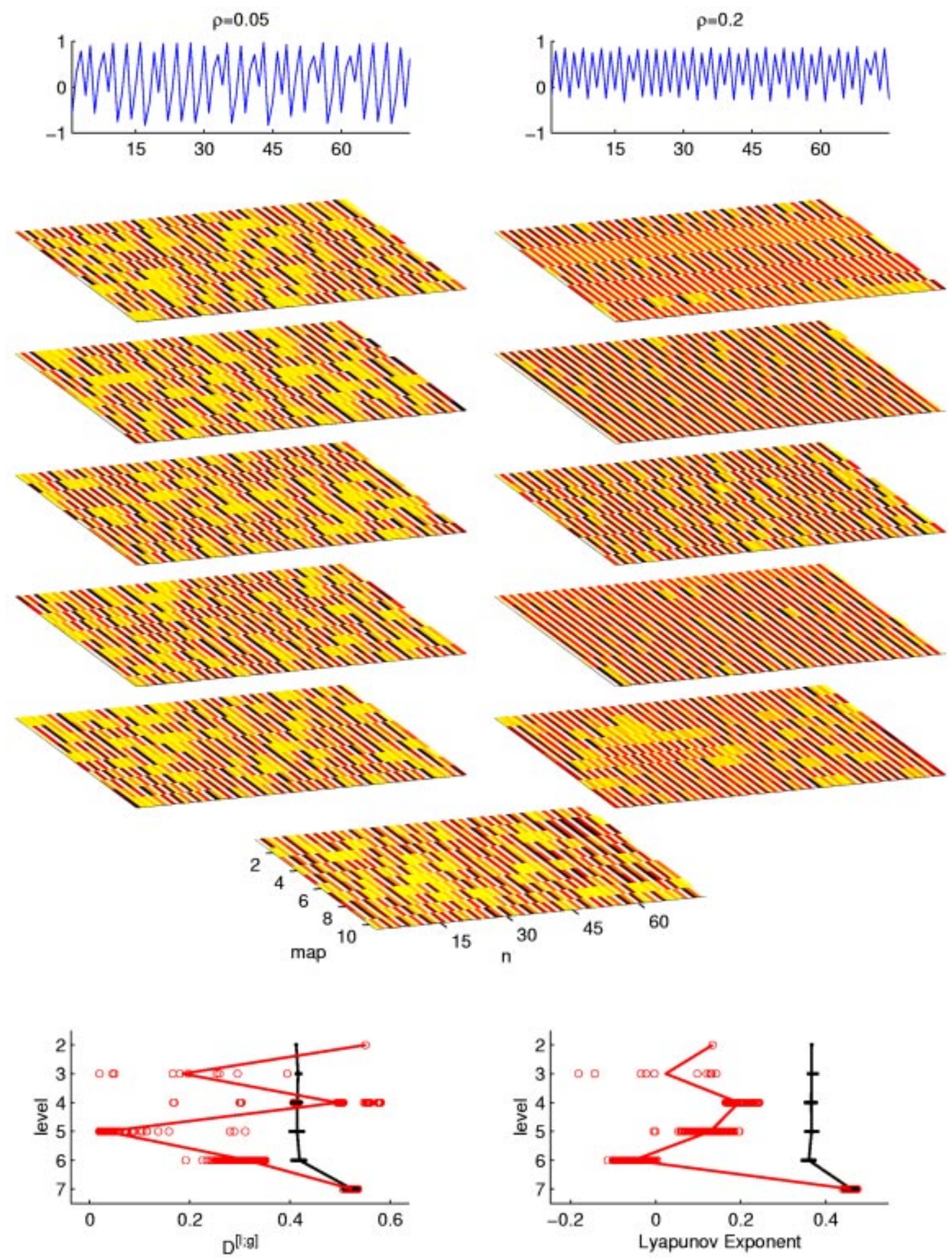

FIG. 8. (Color online) Hierarchies with 7 levels, $N=10$ maps in each block, $a=2$ and $\epsilon=0.1$. The shadings show representative lattices from the lower levels (the color map is chosen to emphasize differences amongst the elements); the top picture shows time series of the presiding map at the pinnacle of the hierarchy. The left-hand panels have $\rho=0.05$, and those on the right have $\rho=0.2$. The two pictures at the bottom of the collage show (a) the normalized squared differences, $D^{[l ; g]}$, and (b) the leading Lyapunov exponents for lattices at each level, $l$. The points show the values of the two quantities for each group, $g$, and the lines indicate the average over all groups on the same level. In each of the panels, we show the two cases displayed in the remainder of the figure, dots are for the case on the left; circles are for that on the right.

squared differences, clusters somewhat smaller, but nevertheless appreciable differences, and nearly synchronized solutions have negligible ones). The Lyapunov exponents highlight how the base of the hierarchy at these parameter values contains a variety of both periodic and chaotic clusters. In the case with weak vertical coupling, positive exponents further up the hierarchy indicate the independent evolution of those levels; the other case, with stronger vertical coupling, 

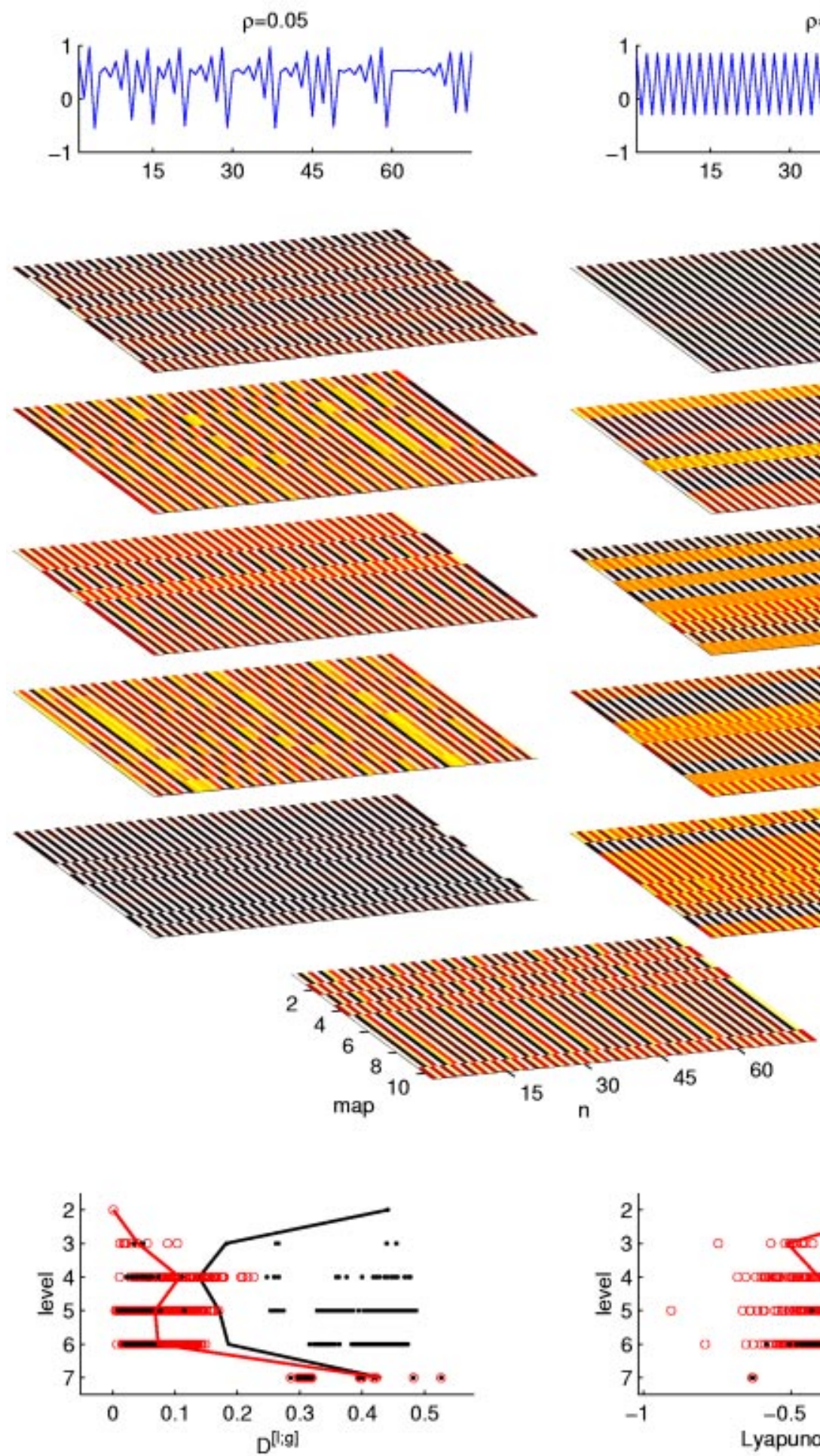

FIG. 9. (Color online) A similar picture to Fig. 8, but for $a=1.7$ and $\epsilon=0.2$. The left-hand panels have $\rho=0.05$, and those on the right have $\rho=0.5$.

is, however, slaved to the base level and has "noisy" periodicity-rough periodic motion with low-level noise. The spread in the values of both $D^{[l ; g]}$ and the Lyapunov exponents reflects the presence of a large number of different kinds of cluster states at most levels.

(3) $(\epsilon, a)$ in the chaotic synchronization regime: For small $\rho$, the lowest levels are in a state of imperfect synchronization; each element follows a similar path interrupted by episodic desynchronizations. As we raise $\rho$, the amount of
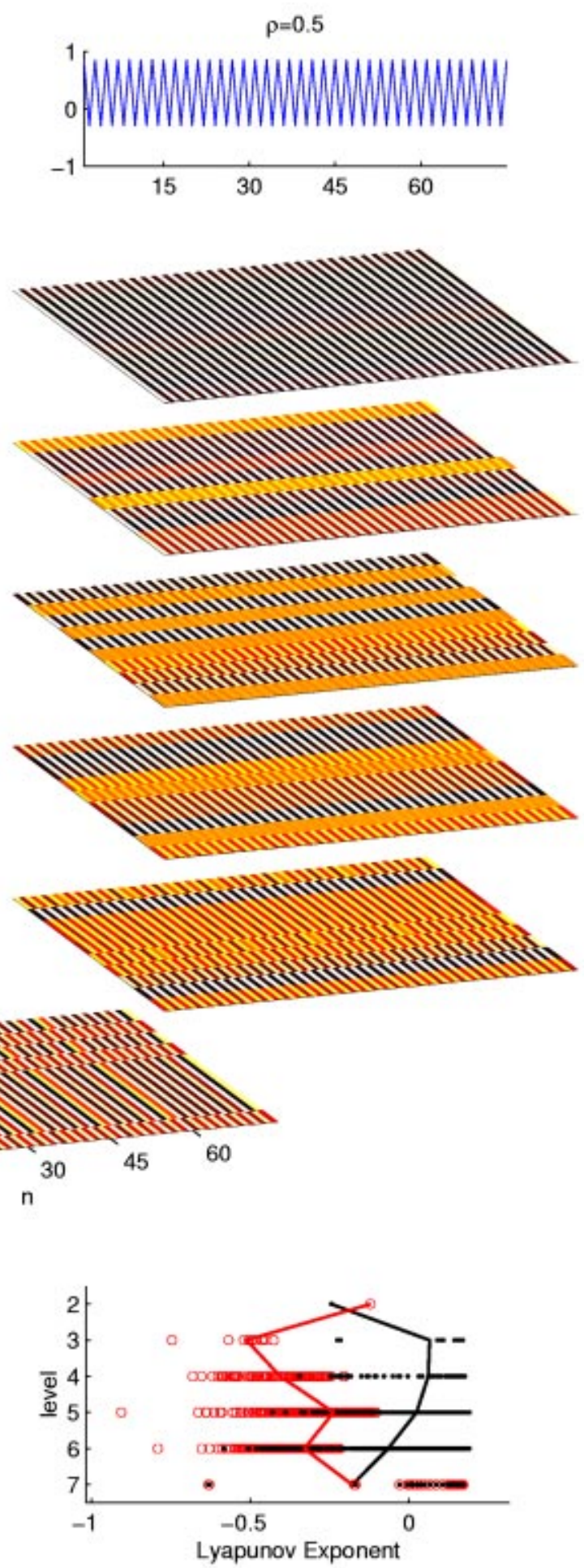

Lyapunov Exponent 
(a)
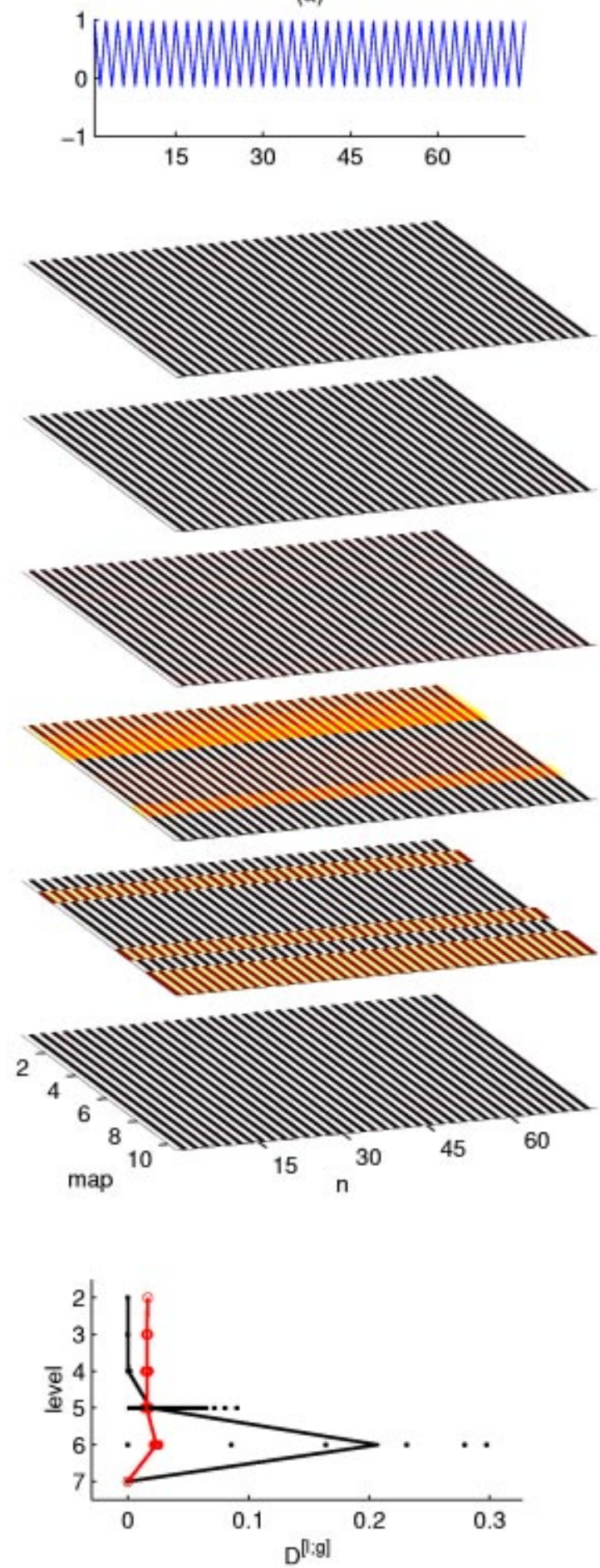

(b)
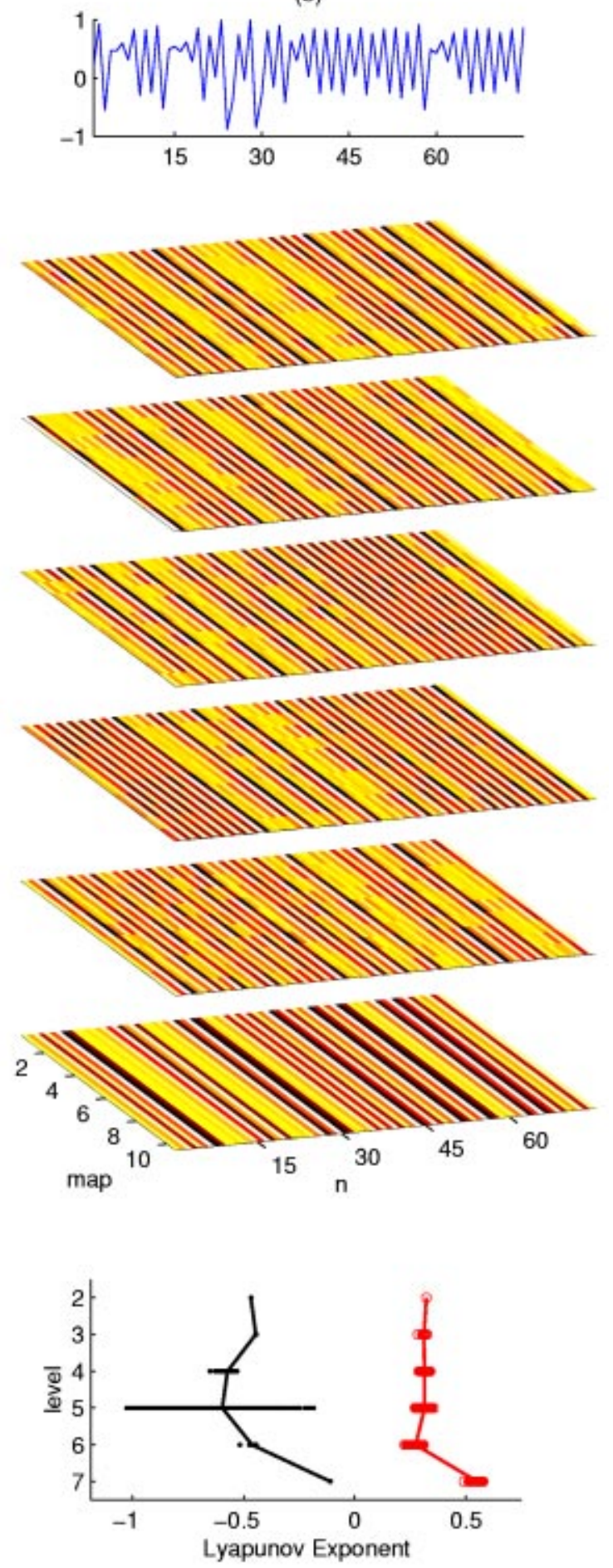

FIG. 10. (Color online) A similar picture to Fig. 8. The left-hand panels have $a=1.2, \epsilon=0.25$, and $\rho=0.3$, and those on the right have $a=1.9, \epsilon=0.5$, and $\rho=0.1$.

These two final cases are illustrated in Fig. 10. The series on the left displays a hierarchy with a periodic, synchronized lowest level; clusters are obvious at all levels and the top map is a period- 2 orbit. The series on the right shows imperfect synchronization. The emergence of periodic clusters in the first case is clearly revealed by the spread of non-negligible squared differences at the fifth and sixth levels and the negative Lyapunov exponents. Surprisingly, there is some suggestion that the periodic hierarchy converges to a self-similar state at the higher levels, despite the pronounced clustering at the lowest levels.

Note that, because of the constraint $1>\epsilon+\rho$, it is not always possible to sample all the ranges of behavior as we fix $(\epsilon, a)$ and vary $\rho$ (for example). Also, exceptional cases occur if there are equally partitioned, period-2, two-cluster states; such states have constant mean field and so their ef- 
fect on the level immediately above does not fall into the standard pattern.

\section{E. Discussion}

Given our "rules of thumb" for understanding the dynamics, we can now return to the questions posed in the Introduction: Disorganization at the lowest level can indeed be organized further up the hierarchy; this is the renormalizing effect of a constant mean field. The converse is also true: the complexity arising when levels can cluster and choose different phases of a periodic orbit destroy the ability of the hierarchy to continue to cluster at the higher levels. To choose among the possibilities, we must be sensitively aware of the values of the coupling strengths and the map parameter; Secs. IV B and IV C offer criteria indicating whether a lattice begins to organize or whether the whole system is incoherent.

Because organization can occur as we transcend levels in the hierarchy, the temporal complexity need not increase with the number of levels. Indeed, only two of our sample hierarchies show leading Lyapunov exponents that become independent of level (Figs. 8, left, and 10, right). These two cases are examples of "extensive systems," in which the degree of complexity (as measured, for example, by the number of positive Lyapunov exponents in each group) scales with system size, and are therefore hierarchies with self-similar dynamics. Curiously, these examples have parameter values from different extremes of the parameter ranges: $\rho$ and $\epsilon$ both small and $\rho$ and $\epsilon$ both (relatively) large, respectively. The first case is what one might call "fully developed incoherence," and all the maps have a degree of independence; the characteristic measures scale with map number. In the second case, the maps within each block are strongly synchronized, and characteristic scaling occurs with block number, not element number. Hence, only the blocks are independent, and scaling is much weaker.

Of the other sample hierarchies, one is purely periodic at all levels (Fig. 10, left) and another has positive exponents at only the base level (Fig. 9, right). The lack of an increase in temporal complexity on escalating through the second hierarchy reflects how the higher levels do not evolve independently. In the chaotic case, the upper levels are almost periodic, and disturbed by a low-level noise that is really the high-dimensional signature of the lowest chaotic level. Thus, such lattices illustrate the vision in which unresolved, highdimensional dynamics can be modeled as noise. Even if the dynamics is not actually chaotic, but periodic at all levels $\left(a<a_{\infty}\right)$, the hierarchy has a different form of complexity due to the extreme multiplicity of distinct attractors. This is the generalization of the "attractor crowding" described by Weisenfeld and Hadley. ${ }^{9}$

We may also use our guidelines to predict how the mapnumber per lattice $N$ influences the dynamics of the hierarchy. Evidently, when maps in each group lack coherence or are closely synchronized there can be little change on varying $N$. However, in hierarchies where organization begins on escalating through levels, by adding further maps to each group we can multiply the varieties of different driving signals entering a given level from below. Hence, true clustering becomes an even more remote possibility and the quality of imperfect clusters can degrade as we raise $N$. Nonetheless, the phenomenology we have described remains the same, and we have verified this numerically by varying $N$ in the computations.

Lastly, we stress again that the present results refer to a lattice where the coupling is unidirectional, from lower to upper levels. The breaking of this asymmetry can drastically change the behavior, leading to full synchronization and, presumably, to a still richer ensemble of dynamical regimes.

\section{CONCLUDING REMARKS}

Loosely speaking, in biological research an understanding of the workings of living organisms is gradually built up by taking molecular units and combining them into larger units, then taking the new, integrated units and combining them into even larger components, and so on to create a rich, complicated hierarchical structure. Jacob ${ }^{10}$ called the entity formed via this integrative procedure, an "integron." Moreover, because of the self-similarity of the construction procedure, one can think of the integron as being assembled from integrons of the level below it, and each integron takes part in the construction of the integron of the level above.

The hierarchical integrating process is not bound to biology but is a metaphor for the organization of many different systems. The contexts range from sociology to physics, in which smaller entities are put together to build up a bigger "organism." For example, in visions of developed turbulence in the physical sciences, eddies of all scales exist, and are fed by an energy cascade from the large to the small scale. It may be interesting to use a simple model like the one considered here to explore the reliability of concepts such as eddy viscosity and mean field descriptions, that can become ill-defined when the lower levels are not totally disorganized. Another example comes from the climate system, that is composed of many interacting units, which are in turn composed of smaller subunits, having, in this case, different time and space scales. In this framework, an interesting question is whether we can approximate the dynamics of the head, or of some of the upper levels, by a coarse-grained description complemented by a stochastic process parameterizing the dynamics of the lower levels. Again, organization in the lower levels may destroy such an attempt.

Of course, any such application is plagued by the requirement to specialize and complexify for the sake of realism; elements of the hierarchy must be designed to suit the problem and their attributes typically vary with the level of integration, breaking the self-similarity, and information is passed amongst levels in a far more intricate fashion than a directed feed from one level to the one immediately above (to name but two of an infinitude of possible complications). Our objectives here have been far more idealistic; our goal was to build one of the simplest mathematical models of a perfect integron. Maps were our "subjects" and we studied their collective behavior within the hierarchically coupled lattice. In this endeavor, we decided to accept some major limitation in the model we used; though it is true that hier- 
archies are typical for biological systems and for many other natural systems, one should not forget that different levels in natural hierarchies are characterized by different time scales. In many instances, this property is crucial. The model investigated here lacks this property, and one should be aware of this limitation in applications and extensions to real biological and sociological systems. A second important limitation comes from the fact that the coupling in the model is unidirectional, and the upper levels in the hierarchy cannot feed back on the lower levels. Notwithstanding these limits, we hope that the mathematical game in which we have indulged will prove useful to those dealing with the complicated hierarchies encountered in physical and biological applications.

\section{ACKNOWLEDGMENTS}

R.S. was supported by the Isabella Bonadonna 1998 fellowship from the Associazione Elettrotecnica ed Elettronica Italiana (AEI). He also acknowledges useful conversations with Maria Gabriella Signorini in the early stages of the work. We thank Ed Spiegel for important advice at the beginning of this work, but we pressed on anyway.
${ }^{1}$ Strictly speaking, our fundamental element is a "quadratic map" rather than the "logistic map," but the two are equivalent upto a scaling. The logistic map is defined through $\xi_{n+1}=r \xi_{n}\left(1-\xi_{n}\right)$. After the change of variables $x=(4 \xi-2) /(r-2)$ and $a=r(r-2) / 4$, we return to the quadratic map. The transformation is continuous and invertible for $r=(2,4)$.

${ }^{2}$ K. Kaneko, "Overview of coupled map lattices," Chaos 2, 279 (1992).

${ }^{3} \mathrm{~K}$. Kaneko, "Clustering, coding, switching, hierarchical ordering, and control in network of chaotic elements," Physica D 41, 137 (1990).

${ }^{4}$ N. J. Balmforth, A. Jacobson, and A. Provenzale, "Syncronized family dynamics in globally coupled maps," Chaos 9, 738 (1999).

${ }^{5}$ T. Shibata and K. Kaneko, "Tonguelike bifurcation structures of the mean-field dynamics in a network of chaotic elements," J. Phys. D 124, 177 (1998).

${ }^{6}$ The lack of true synchronization is related to the purely bottom-up coupling of the system considered here. Adding a further top-down coupling from upper to lower levels may lead to true synchronization of the system. However, we decided not to explore the even more complex dynamics of a fully coupled hierarchy.

${ }^{7}$ S. C. Venkataramani, B. R. Hunt, and E. Ott, "Bubbling transition," Phys. Rev. E 54, 1346 (1996).

${ }^{8}$ G. Benettin, L. Galgani, A. Giorgilli, and J.-M. Strelcyn, "Lyapunov characteristic exponents for smooth dynamical systems and for Hamiltonian systems: A method for computing all of them. Part I: Theory, and part II: Numerical application," Meccanica 15, 9 (1980).

${ }^{9} \mathrm{~K}$. Weisenfeld and P. Hadley, "Attractor crowding in oscillator arrays," Phys. Rev. Lett. 62, 1335 (1989).

${ }^{10} \mathrm{~F}$. Jacob, The Logic of Life: A History of Heredity (Princeton University Press, Princeton, 1993). 